Fecha de recepción: abril 2018

Fecha de aceptación: noviembre 2018

Versión final: diciembre 2020

\section{La transición urbana y social hacia un paradigma de movilidad sostenible}

Gabriela Nuri Barón *

Resumen: El Diseño para la Transición es una disciplina que propone la formulación de visiones de futuro que sean el resultado de innovación local ascendente en constante evolución. En relación a la problemática de movilidad urbana, la sostenibilidad se logra mediante las elecciones de estilos de vida que han sido impulsadas por la visión, al crear espacios de debate acerca de nuevas formas de ser y de hacer, y mediante el co-diseño estrategias para materializar esa visión.

En el ámbito de la movilidad sostenible, la innovación no necesita ser de índole material, sino que resulta de la gestión eficiente, inteligente y participativa de los recursos. Es radical en sus concepciones acerca del bienestar, que se alejan de las propuestas por el mercado y se acercan a aquellas innatas a las personas. Se trata de una movilidad a escala humana, centrada en el usuario no sólo a nivel de escala física, sino a escala emocional y cognitiva. El siguiente trabajo presenta una revisión bibliográfica sobre la movilidad como actividad trasversal a todas las actividades urbanas, proponiendo la bajada a la escala humana como estrategia para la transición. Finalmente se propone un escenario viable de movilidad sostenible donde el rol del diseñador como operador cultural se vuelve fundamental.

Palabras clave: movilidad sostenible - espacio público - caminata - ciclismo - innovación social - diseño participativo - bienestar urbano.

[Resúmenes en inglés y portugués en la páginas 171-172]

(*) Gabriela N. Barón. Especialista en Diseño para la Sustentabilidad e Innovación Social. Diseñadora Industrial graduada en Argentina, realizó un master en "Diseño de Sistemas de Productos y Servicios" en el Politécnico de Milán con una beca al mérito. Posteriormente ha participado en proyectos de conservación ambiental y social en Argentina, Italia y Asia. Actualmente se dedica a la investigación y docencia en Mendoza, en el marco de su beca CONICET de Doctorado en Ingeniería Ambiental. Es profesora de la materia "Diseño para la sostenibilidad y la Innovación social" en Mendoza y dicta cursos de posgrado en movilidad sostenible. Sus líneas de investigación son Accesibilidad y movilidad activa, Huella de Carbono, Diseño de espacios públicos, Design Thinking y Diseño inmaterial. 


\section{El camino de vuelta desde el transporte a la movilidad}

La caminata es una acción humana básica y natural, de hecho es la forma más equitativa de desplazamiento y sin embargo, no ha sido considerada como el modo medular para la planificación del transporte en la mayoría de los proyectos urbanos del último siglo. Este simple hecho ilustra la profunda crisis enfrentada por los estudios tradicionales debido a su foco en soluciones técnicas que perdían de vista el sujeto para el cual planificaban: las personas. Más precisamente: las personas en contexto.

El transporte ha sido históricamente analizado desde enfoques cartográficos y/o técnicos, donde el objeto de estudio es el viaje y la aproximación es material. Estos estudios cuantitativos están frecuentemente enfocados en sujetos como el sistema de transporte en sí mismo, la influencia urbana en patrones de transporte y las políticas económicas que influyen al transporte, entre otros. Del mismo modo, encontramos estudios predominantemente cualitativos que analizan el transporte desde una perspectiva sociológica, en base a las características económicas y culturales del individuo y su influencia en las elecciones de transporte. Asimismo, los estudios de accesibilidad podrían clasificarse según su enfoque, ya sea top-down (descendente) o bottom-up (ascendente). El primero se refiere a la influencia de la forma urbana o las regulaciones en los patrones de movilidad, y el segundo describe cómo los factores individuales predominan en las elecciones modales. Ambas perspectivas son válidas, sin embargo, suelen ofrecer una visión limitada de la problemática ya que las decisiones individuales estarán influenciadas por una combinación de variables de ambos enfoques.

Alrededor del año 2000 comienzan a surgir nuevas metodologías en la bibliografía publicada, ampliando el foco hacia interpretaciones inter-disciplinarias de la problemática. Se produce un cambio de paradigma ilustrado por el descarte del término transporte a favor del término movilidad, donde el campo de estudio es el mismo (el movimiento de la gente en el territorio) pero sus límites tradicionales se han expandido, y en vez de representar visiones opuestas, presenta visiones complementarias. Su convergencia, el área donde el espacio, tiempo, los objetos, los servicios, la comunicación y las emociones se unen, está mejor representada por disciplinas experienciales. Miralles y Cebollada (citados por Hernández, 2012, p. 119) intentan resaltar el hecho de que movilidad y transporte son sólo medios de satisfacer necesidades y no son fines en sí mismos. Asimismo, Gutiérrez (2012) enuncia que el transporte es un aspecto de la movilidad necesario pero insuficiente, ya que no describe la mediación entre sociedad y territorio. Finalmente, Hernández (2012) enfatiza la noción de potencialidad, indicando que los estudios de accesibilidad deberían considerar más allá de los desplazamientos conocidos u observados. De hecho, la mayoría de las encuestas realizadas en América Latina en la última década (Miralles-Guasch, 2012) han sido criticadas por su visión limitada al no haber considerado los viajes no-hechos o potenciales.

La revisión de bibliografía en la temática, evidencia la necesidad de estudiar el transporte humano como problemática trasversal a todas las actividades humanas, dejando atrás paradigmas reduccionistas/mecanicistas, inadecuados para la comprensión de sistemas complejos naturales (Capra y Luisi, 2014). Al mudar el foco del transporte a la movilidad salen a la luz estudios multidisciplinarios, sistémicos y centrados en la persona, que prometen salvar vacíos académicos y proyectuales. 
En sistemas de movilidad modernos, los modos activos como la caminata y el ciclismo son considerados un recurso tangible y gestionable, no sólo para distancias cortas, sino como modos complementarios al transporte motorizado, ya que cada viaje comienza y finaliza con segmentos de acceso activos. Es por esto que la facilidad de flujo a través de espacios de acceso y transición se vuelve un gran determinante de elección de Transporte Público (TP) como modo de movilidad, especialmente en un contexto político-ambiental de desaliento del vehículo particular (Hsiao et al., 1997; Murray et al., 1998).

En la escala urbana, la calidad del espacio público juega un rol fundamental para la movilidad peatonal. Hay entornos que favorecen e invitan a este modo de transporte, fortaleciendo comunidades y revitalizando el ambiente urbano. Los ambientes peatonales se definen como áreas donde predominan los viajes a pie como consecuencia de factores que promueven este modo (Borst et al., 2009; Zacharias, 2001). La presencia o ausencia de elementos específicos a lo largo del viaje, así como sus características morfológicas, puede potenciar o disuadir patrones de movilidad (Valenzuela-Montes y Talavera-García, 2015). En síntesis, se podría decir que la movilidad es una interpretación más amplia del fenómeno de transporte que incluye la dimensión material (cartografía, infraestructura y oferta de servicios), la dimensión socio-económica (Lévy, 2001; Kaufmann, 2002; Orfeuil, 2004) y la dimensión experiencial (Valenzuela-Montes and Talavera-García, 2015).

\section{Accesibilidad: La escala humana de la movilidad urbana}

El término accesibilidad es ampliamente usado para expresar diferentes facetas de la movilidad. En relación a las teorías de necesidades y satisfactores de Max-Neef (1992), la necesidad de desplazarse desde un punto A hasta un punto B será "satisfecha en un manera única en relación a la era, cultura, ubicación geográfica, edad y mentalidad” del individuo. Asimismo, factores de usos del suelo (como la ubicación de las actividades) y las opciones substitutas de movilidad (como las telecomunicaciones y los servicios de delivery) influirán en la necesidad de desplazamiento en sí misma (Litman, 2008).

A lo largo de este trabajo, sin embargo, el término accesibilidad empleado será aquel propuesto por Gutiérrez (2010) que especifica la diferencia entre acceso y accesibilidad. Acceso se refiere a la posibilidad de concretar las necesidades y deseos que motivan el viaje, mientras que accesibilidad se refiere a la facilidad de realizar el viaje. El concepto de accesibilidad como la facilidad para realizar viajes activos, no-motorizados, evidencia una multiplicidad de factores que afectan a la movilidad en la escala humana. En este marco contextual, la accesibilidad está directamente influenciada por las características materiales y semióticas específicas al camino recorrido, que resultarán en experiencias individuales que afectarán directamente las elecciones modales (Ver Figura 1).

\section{Las calles como una red interconectada de espacios públicos}

En el ámbito de movilidad, la dicotomía privado $v s$. público posee dimensiones políticas profundas que han sido fuente de fuertes debates. Desde una perspectiva sociológica, Hernández (2012) mide accesibilidad como el grado de correspondencia entre las oportunidades de movilidad (provistas por los servicios y la infraestructura) y los recursos de los 
hogares para acceder a ellas. Similarmente, Miralles y Cebollada (citado por Hernández, 2012, p. 119) definen la accesibilidad como la facilidad de los ciudadanos para cubrir la distancia que separa dos lugares, y la subsecuente capacidad para ejercer sus derechos ciudadanos de movilidad.

Sin embargo, el debate de público/privado en movilidad no sólo se refiere al acceso al servicio sino al uso del espacio mismo.

Los espacios públicos son todos los lugares de propiedad o acceso público, accesibles y disfrutables gratuitamente por todos los ciudadanos, sin fines de lucro. Pueden consistir de espacios abiertos (calles, aceras, plazas, jardines, parques, etc.) o de espacios cubiertos (bibliotecas, museos, etc.). (Charter de espacio público, 2013)

Estos espacios deben ser físicamente accesibles por todos los ciudadanos promoviendo la diversidad total, la inclusión y la versatilidad de funciones.

La equidad en el uso del espacio público constituye un fuerte argumento de la crítica a las ciudades auto-céntricas. Bajo esta perspectiva, las calles se consideran como el espacio público por excelencia, ya que ocupan un área urbana extensa y distribuida, formando redes de espacio accesible interconectado, constituyendo la base espacial de los intercambios sociales y económicos urbanos.

La disciplina del placemaking (hacer lugares) equilibra el diseño urbano descendente, o top-down, mediante la propuesta de iniciativas pequeñas, conectadas, ascendentes o bottom-up, participativas y centradas en las personas. "El placemaking promueve un principio simple: si planificas las ciudades para los automóviles y el tráfico, obtienes automóviles y tráfico. Si planificas para personas y lugares, obtienes personas y lugares" (PPS, 2012). El proceso de placemaking resignifica las calles como destinos en sí mismas y no solamente espacios de transición. Los lugares vibrantes y placenteros construyen democracia a través de procesos participativos nacidos de la comunidad y fortalecidos mediante alianzas estratégicas con actores locales. Sumado a esto, los espacios públicos activos realzan las interacciones sociales resultando en mejoras a nivel seguridad, salud pública y compromiso cívico. La co-creación permite la apropiación de los espacios comunes, habilitando instancias de interacción repetida, versatilidad de uso y continua re-invención urbana. Un excelente ejemplo de los resultados de políticas de placemaking es la ciudad de Medellín en Colombia (Ver Figura 2).

\section{La Experiencia de Usuario como fuente de datos cualitativos}

El término User Experience (UX) proviene del campo del diseño de servicios. El diseño de servicios es una ciencia multidisciplinaria que ha ganado popularidad en su aproximación a la empresa moderna, la investigación en diseño y la educación en el diseño. Con la popularización del Design Thinking (Martin, 2009) el diseño de servicios se afianza validando metodologías para la colección de datos cualitativos mediante análisis de experiencia de usuarios. Bajo la perspectiva del diseño urbano a escala humana, surge la necesidad de comprender las etapas pre y post viaje del transporte como un servicio. Allí es cuando la disciplina de 


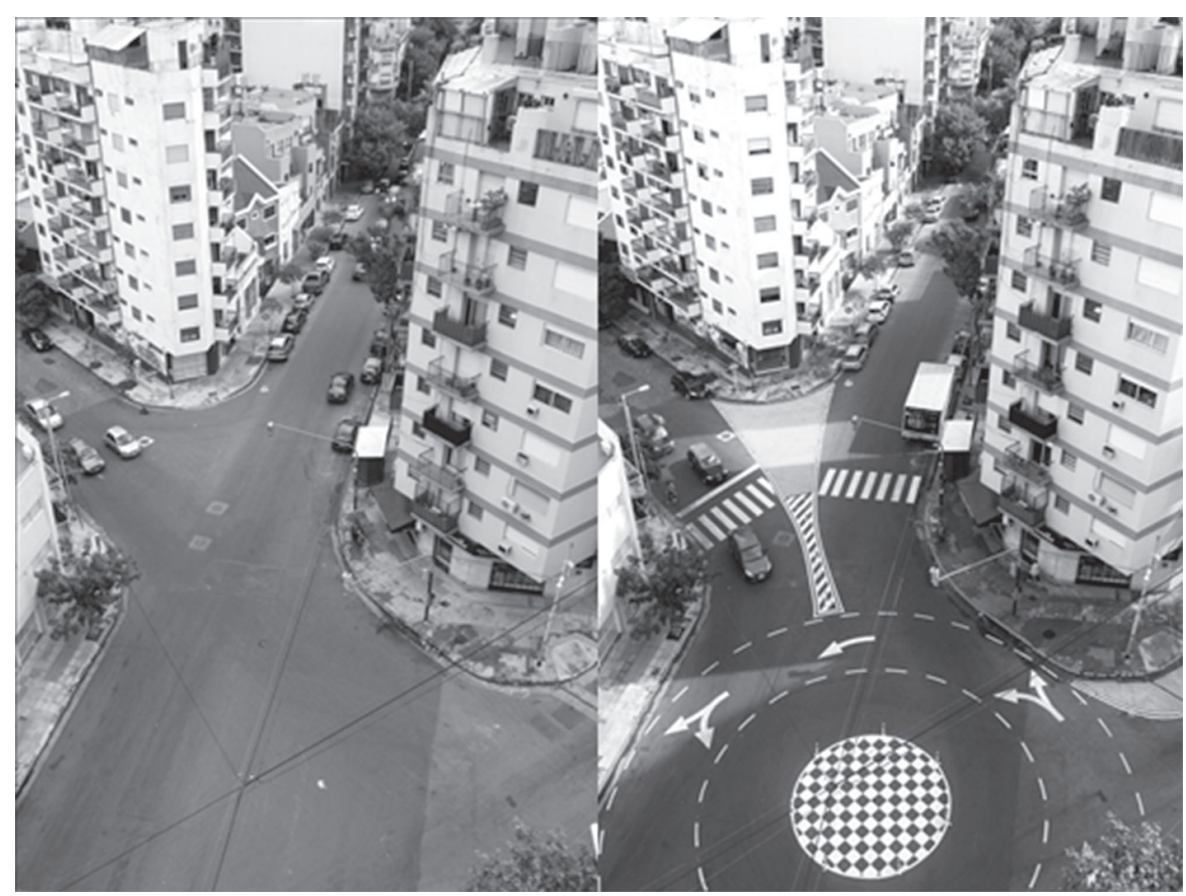

Figura 1.

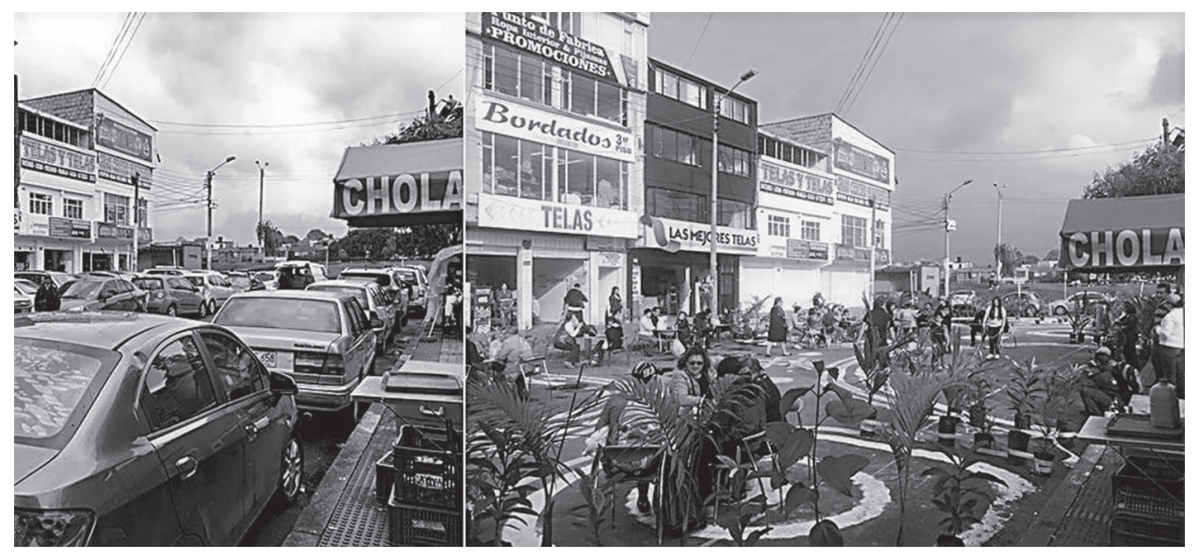

Figura 2.

Figura 1. Reducción del espacio automotriz a favor de una escala humana. Imagen de una intersección en la ciudad de Buenos Aires antes y después de la intervención. Fuente: Bisiau, 2014. Figura 2. Placemaking en Colombia. Imagen de antes y después de intervención de estacionamiento en espacio público. Fuente: Brent Toderian (en Twitter). 
UX provee un marco válido para describir cualitativamente estas circunstancias, esto es, lo que la gente hace, siente y piensa. Los viajes de cliente descriptos por Miettinen y Koivisto (2009) se basan en la premisa de que los servicios son procesos que se desarrollan en el tiempo y se componen de momentos. Cuando se conectan todos los momentos de un servicio, se forma un viaje de cliente, a partir de las acciones explícitas del proveedor y las decisiones del usuario.

En el campo de la movilidad, la UX se ha analizado mediante el uso de herramientas participativas diseñadas para comprender la ecología de las experiencias. Un buen ejemplo es el trabajo de Culén et. al. (2014) que propone un set de tarjetas de experiencia de viaje con el fin de obtener respuestas ricas por parte de los usuarios de un servicio de TP. Las tarjetas permiten describir aspectos experienciales desde las etapas pre viaje (como la planificación) hasta el arribo a destino (Ver Figura 3).

\section{Accesibilidad en el ambiente urbano: un esfuerzo multidimensional}

De acuerdo a Valenzuela-Montes y Talavera-García (2015) es necesario comprender mejor los factores que favorecen la caminata como modo de transporte urbano, y como modo de acceso a sistemas públicos de transporte. Estos autores han realizado un exhaustivo estudio bibliográfico clasificando publicaciones científicas en base a una visión de escala humana. Se distinguen tres aproximaciones: el enfoque peatón-transporte que se basa en la caminata como modo de transporte en sí, donde el viaje posee un origen y un destino, se refiere a la dimensión funcional y posee variables tales como "distancia". Complementariamente, el enfoque peatón-entorno le asigna mayor relevancia a las características urbanas del lugar donde se produce el viaje, se refiere a la dimensión morfológica y posee variables como "conectividad". Asimismo, existe un tercer enfoque mixto, que combina aspectos funcionales y morfológicos en una dimensión ambiental, donde se pueden inferir variables como "esfuerzo".

Wardman (1998) introdujo un marco de referencia que reconoce las dimensiones cognitiva y emocional de las personas en relación a la movilidad urbana y que mejor refleja los esfuerzos del diseño a escala humana que postulaba Papanek ya desde los años '70 (Papanek, 1983). Posteriormente, Shakespear (2014) retoma estos conceptos en sus trabajos de señalética para las calles de Buenos Aires. De esta forma, el esfuerzo humano de acceso se puede representar por medio de una variable triple: físico, emocional y cognitivo. Al considerar a la persona desde una visión holística se pueden identificar cualidades experienciales, analizarlas y volverlas a conjugar para comprender cómo éstas afectan la toma de decisiones con respecto a transporte. A continuación se desarrolla en profundidad cada aspecto del esfuerzo humano (Ver Figura 4).

\section{Esfuerzo físico}

El esfuerzo físico es aquel que se consume durante todas las etapas de un viaje, ya sea caminando, esperando o sólo manteniendo la postura corporal (Shakespeare, 2014). Cuando las 

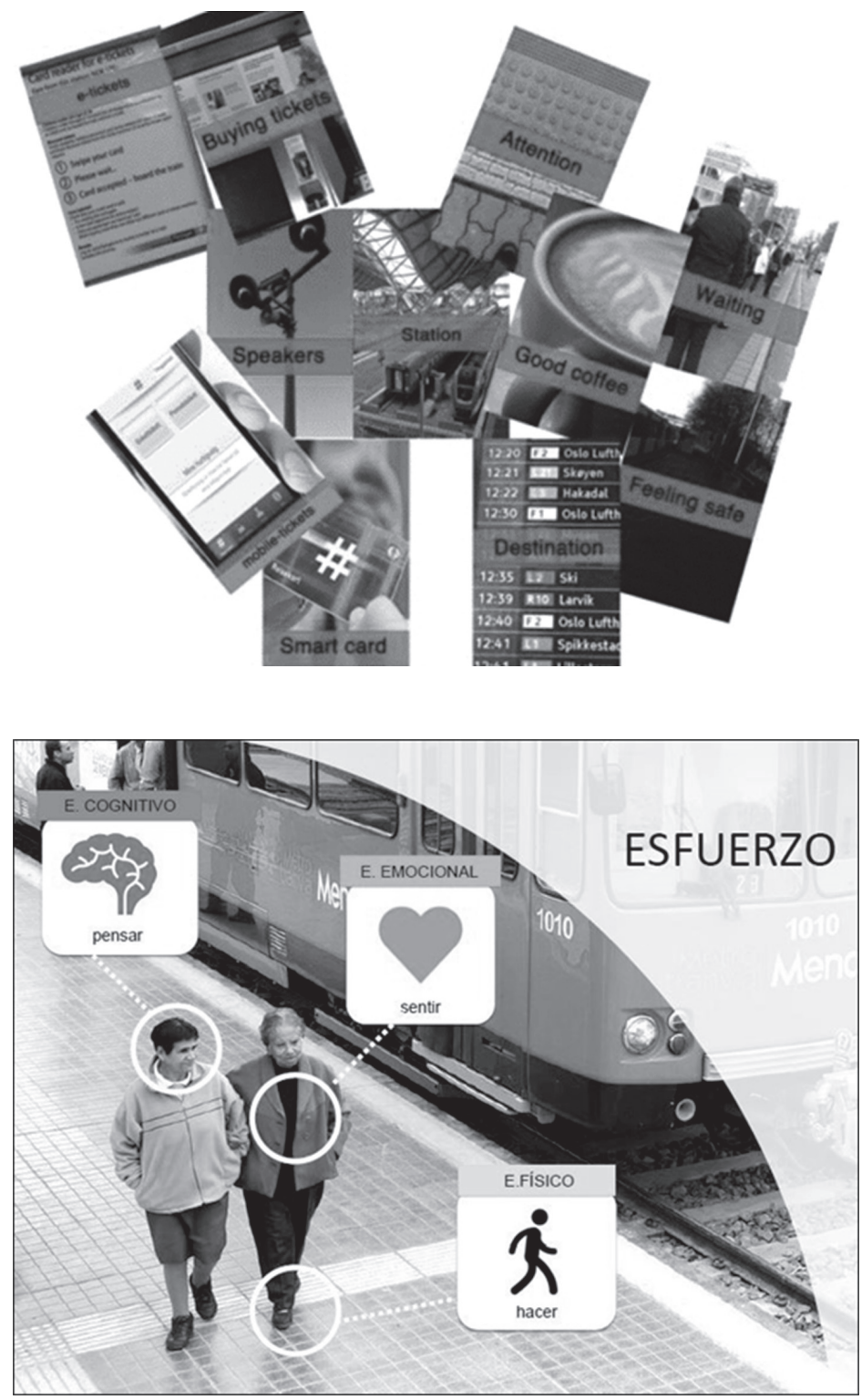

Figura 3. Experiencia de Usuario: Tarjetas que permiten describir los encuentros con elementos de interface del servicio. Fuente: Culén et al. 2014.

Figura 4. El esfuerzo humano se presenta como una variable tridimensional: esfuerzo físico, esfuerzo emocional y esfuerzo cognitivo. Fuente: Barón, G. 2017. 
personas interactúan con el ambiente, aspectos físicos como la topografía, la pendiente y las condiciones climáticas particularmente afectarán las acciones relacionadas a la movilidad. La distancia es el factor más influyente en la decisión de caminar o tomar otro modo de acceso (Valenzuela-Montes y Talavera-García, 2015). A pesar de que numerosos autores han calculado las distancias que están dispuestos a recorrer los peatones para acceder a paradas de TP, los resultados varían en relación a la demanda, la calidad de la superficie, la redundancia del servicio y los aspectos culturales y físicos de las personas. Así mismo, la forma urbana determina la conectividad de la grilla, en relación a la cantidad de intersecciones viales que comprende un área buffer de captación, lo cual influye en la distancia a recorrer. Del mismo modo, factores atmosféricos como la temperatura y el clima influirán en la facilidad de acceso (Kuby et al., 2004; Lam y Morrall, 1982). Sin embargo, los estudios en la temática no son conclusivos al establecer una relación entre el clima y la disposición a caminar o viajar en bicicleta, ya que los casos de estudio con mayores poblaciones que eligen estos modos como Amsterdam o Copenhague poseen un clima notablemente más riguroso que otras ciudades con climas más adecuados para estos modos (y menor incidencia) como San Francisco (Pucher y Buehler, 2008).

\section{Esfuerzo emocional}

Este factor afectivo se refiere a la energía emocional consumida durante el viaje en relación a la incertidumbre de viajar de forma segura y confortable, así como la llegada a tiempo a destino (Shakespeare, 2014). Se debe considerar que desde el punto de vista del individuo la ciudad no es un espacio homogéneo, sino un mosaico de espacios conocidos y desconocidos (Institute pour la Ville en Mouvement, 2013). La persona clasifica los espacios subjetivamente y esta cualificación explica por qué una persona elige una ruta sobre otra, por ejemplo, cuando en vez de elegir el camino más directo se da un rodeo para evitar una zona peligrosa.

La seguridad es un problema con dos facetas: una es el riesgo real de ser víctima de un ataque o accidente y la otra es el temor a ser una víctima. La segunda es llamada seguridad percibida y es la emoción que influirá en las decisión modal y de trayecto (Gomez, 2000). Mientras que esta problemática afecta a todos los grupos sociales, los más vulnerables son aquellos que no poseen una alternativa modal y deciden evitar el viaje y cesar las actividades asociadas. En la mayoría de los casos estas son actividades fundamentales, como trabajo o estudio, causando gran impacto en la economía familiar de estos grupos vulnerables (World Bank, 2002).

Seguridad Civil. Al hablar de seguridad civil nos referimos a la vulnerabilidad de los viajantes hacia actos criminales o antisociales (World Bank, 2002). Se trata de un problema social complejo que afecta gravemente a los países en desarrollo sin limitarse solamente al sector transporte. Particularmente, América Latina posee un alto índice de ataques civiles durante circunstancias de transporte no-motorizado (World Bank, 2002).

Es escasa la bibliografía que relaciona numéricamente la seguridad civil con la probabilidad de usar un medio de TP. Generalmente los casos de accidentes y delitos de este tipo no se reportan ni cuantifican, principalmente los actos de violencia personal o acoso sexual. 
Sin embargo, encuestas sociales en América Latina demuestran su ocurrencia frecuente (Gomez, 2000). A nivel individuo es difícil caracterizar la seguridad percibida, al ser ésta una emoción personal que depende de factores internos, altamente influenciada por experiencias previas y medios de comunicación masiva, entre otros.

A nivel urbano, la seguridad ya sea real o percibida, está relacionada con los usos del suelo, el horario del viaje, la densidad poblacional, entre otros. A nivel de infraestructura, factores como la presencia de iluminación y cámaras de seguridad pueden influir la percepción de niveles de seguridad y desalentar el crimen.

Seguridad Vial. En los países en vías de desarrollo, mueren anualmente medio millón de personas y 15 millones sufren accidentes de transporte, siendo en su mayoría peatones y ciclistas (World Bank, 2002). Los peatones son el grupo más vulnerable de transportistas ya que comparten el espacio con vehículos motorizados y están expuestos a accidentes durante las primeras y últimas millas de su viaje en TP (World Bank, 2002).

Las características del ambiente construido influyen directamente en la seguridad vial, variable clave en el fomento de la peatonalización (Fitzpatrick et al, 1997; O'Sullivan and Morrall, 1996; Schlossberg et al., 2007). La provisión de infraestructura adecuada puede proteger a ciclistas y peatones a través de veredas continuas, cruces señalizados, ciclovías, etc. (Schlossberg et al., 2007). En general, está claro que en contextos urbanos, la infraestructura para modos no-motorizados es más equitativa y notablemente más económica que la infraestructura vehicular.

Es importante mencionar que existe una dificultad en Latinoamérica para relacionar los accidentes viales con el ambiente construido, ya que la documentación de incidentes de modos no-motorizados rara vez se documenta o clasifica. Se estima que del 35\% al 88\% de los accidentes no-fatales con peatones no se denuncian (Hook, W. 2005). La razón es que las víctimas temen multas o arrestos, y que las autoridades no están capacitadas para colectar información adecuada y específica acerca de esta modalidad (World Bank, 2002). Mientas que existen escasas metodologías para medir el esfuerzo emocional, el concepto de niveles de stress ha sido ampliamente aplicado en las últimas décadas. Las metodologías de "Levels of Traffic Stress" (Landis et al., 1997; US Federal Highway Administration, 1998; Minetta Institute, 2012) han abierto líneas de investigación valiosas para medir niveles de seguridad percibidos.

\section{Esfuerzo Cognitivo}

Dos tipos de redes son necesarias para que un Sistema intermodal de transporte funcione: las redes físicas y las semióticas. Las primeras se refieren a infraestructura de transporte y sus componentes, las segundas se refieren a redes de comunicación que permitirá que los potenciales usuarios conozcan, comprendan, signifiquen y formen parte de un servicio. El esfuerzo cognitivo depende de la capacidad de recolección y procesamiento de información durante la planificación, navegación (monitoreo del viaje) y corrección eventual de errores (Shakespeare, 2014). La disponibilidad de información adecuadamente codificada es fundamental para la adopción de un sistema de TP (Blair y Burckhart, 2009). 
Thaddeus y Maine (1994) establecen que la etapa de planificación constituye una parte integral del viaje mismo, reconociendo que cada viaje se compone de 3 momentos. Estos son el momento pre-viaje (relacionado con la toma de decisiones, la planificación y la organización), el viaje (desplazamiento material desde origen hasta destino), y el post-viaje (la consumación de la actividad que motiva el viaje). Esta aproximación facilita en análisis de matices experienciales, al colectar datos concretos desde el momento en el que toma la decisión modal y la estrategia de recorrido. En el momento pre-viaje prevalecerán las características socio-económicas de los individuos (Gutierrez, 2012), mientras que en la subsiguiente etapa ejercerán mayor influencia las características del ambiente construido y del servicio de TP.

La disponibilidad de información relativa al viaje influirá en los niveles de stress cognitivo y emocional de los individuos, quienes tendrán cierto nivel de conocimiento previo acerca de sus opciones. En el campo de movilidad se han identificado cuatro tipologías de canales de diferente naturaleza: señales viales, información gráfica estática, información en tiempo real e información virtual (web).

Señales viales. Las señales urbanas poseen la función de guiar a los sujetos y brindarles coherencia durante el trayecto, bajando los niveles de stress y favoreciendo la seguridad vial (Thompson et al., 2013). La señalización de trayectos específica para modos activos es altamente importante, ya que su red de vías segregadas es en general discontinua y requiere de conexiones complejas.

La señalización peatonal ha probado mejorar la experiencia de los peatones al tiempo que favorece este modo como complemento del TP. La comunicación de tiempos medios de caminata (ver imagen) y disponibilidad de conexiones favorece la inter-modalidad, especialmente en ciudades con afluencia del turismo (Ver Figura 5).

Información gráfica. La información gráfica esquemática es probablemente el canal más validado de comunicación entre los sistemas de TP y los ciudadanos. El ejemplo más famoso es probablemente el mapa de metro de Londres de 1931 que consiste en un diagrama lineal codificado por colores. Este mapa no posee una correspondencia geográfica acertada, sino que se sintetiza mediante un esquema abstracto con alto poder comunicacional (que ha sido validado por las neurociencias). Una encuesta conducida por IVM a pasajeros Latinoamericanos revela que el $96 \%$ de ellos consideran necesaria la existencia de diagramas que indiquen ruta, paradas y conexiones. Es más, dada la cantidad de turistas que usan TP, los autores consideran necesaria la adición de lugares de interés con el propósito de crear una relación cognitiva directa entre el espacio público y la movilidad de acceso (Institute pour la Ville en Mouvement, 2013) (Ver Figura 6).

Información en tiempo real. Dado que la movilidad es una acción urbana altamente dependiente del tiempo, la información en tiempo real se ha vuelto una herramienta de imprescindible en sistemas de TP. La disponibilidad de este tipo de información gana importancia en casos de alteraciones del servicio debidas a eventos no programados. Mientras que usualmente se ha implementado mediante el uso de carteles digitales luminosos que anuncian el tiempo de espera al próximo medio (con el propósito de que los usuarios 

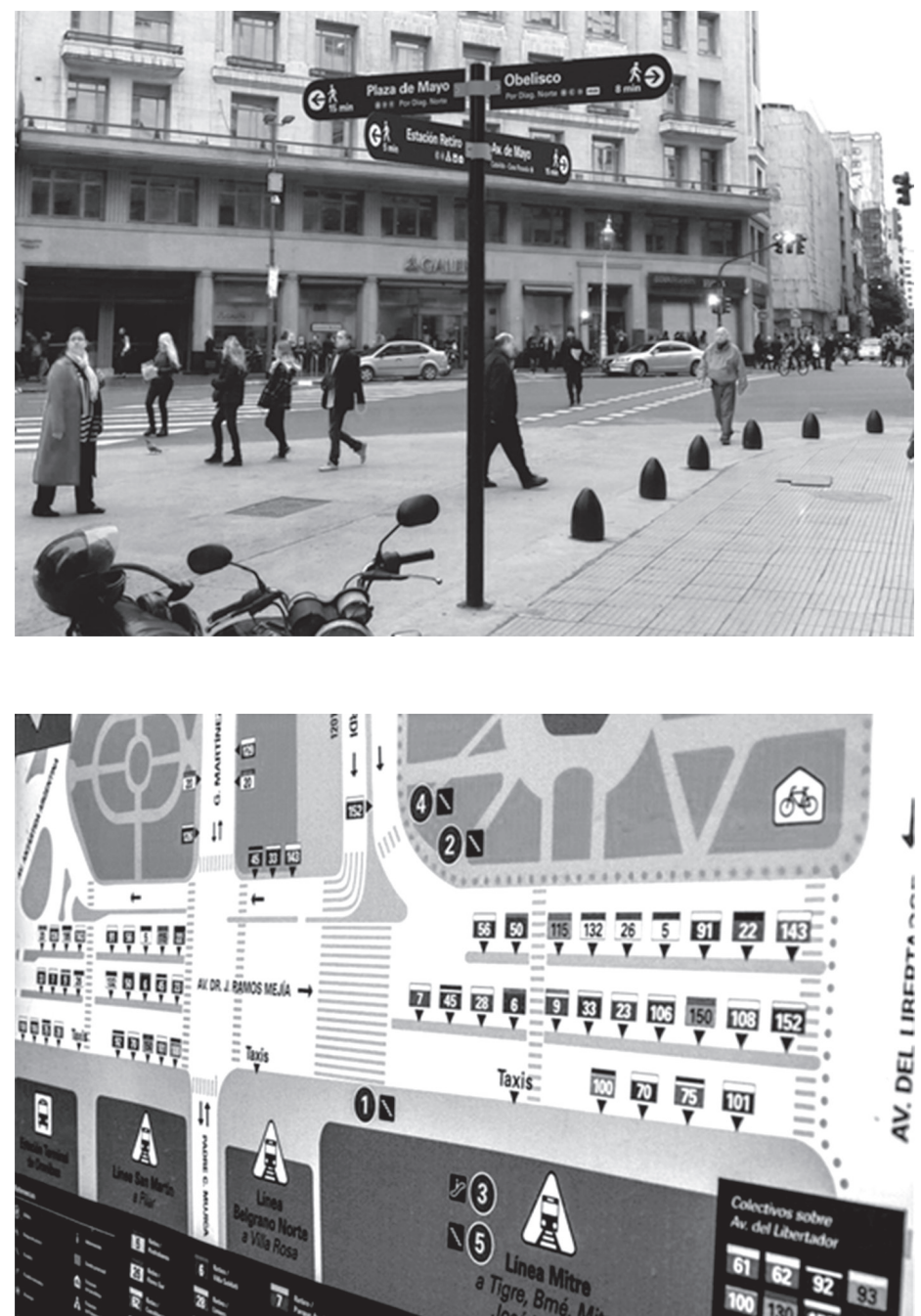
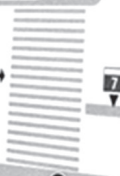

\section{in}

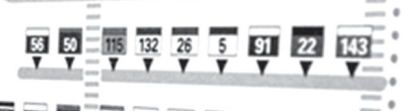

OS
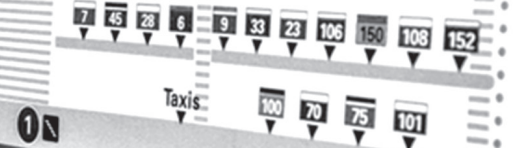
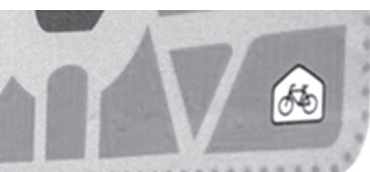

.
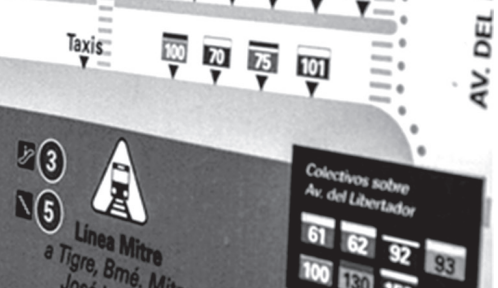

Figura 6. Esfuerzo

cognitivo: Póster backlight que representa un sistema complejo para áreas de trasbordo en Buenos Aires. Fuente: IVM 2014.

puedan cambiar su estrategia de movilidad lo antes posible), actualmente se están utilizando como canal principal las redes sociales accesibles mediante los teléfonos inteligentes de los mismos usuarios.

Información virtual. La extensión de los centros urbanos y sus complejidades en movilidad requieren de la presencia de mecanismos virtuales que representen a esos sistemas 
de modo comprensible (Institute pour la Ville en Mouvement, 2013), reduciendo así la complejidad urbana. La presencia de páginas web oficiales que brindan información de servicios de transporte está creciendo rápidamente en la web. Complementariamente, herramientas cartográficas virtuales como Google maps, accesibles en las etapas de pre-viaje y durante el viaje mismo han mejorado simplificado significativamente las experiencias de movilidad para aquellos individuos que poseen acceso a internet.

\section{La transición urbana y social hacia una movilidad centrada en las personas}

Finalmente, surge la pregunta indispensable: ¿Cuáles son los pasos de la necesaria transición hacia un paradigma de movilidad sostenible?

Profesionales de diversos ámbitos han encarado la tarea de desarrollar alternativas más sostenibles de movilidad en escalas personales tanto como regionales. En línea con la ampliación del rol profesional del diseño, que lo fuerza a evolucionar desde la modelación de aspectos materiales a aspectos inmateriales, diferentes niveles de proyecto han sido abordados desde el diseño de productos hasta el diseño para la innovación social (que trabaja en las esferas culturales y filosóficas) desde propuestas tímidas hasta innovaciones radicales. En base a los abordajes de Tukker (2004) y Vezzoli (2007) se presenta a continuación una interpretación personal de la problemática:

\section{a. Innovaciones incrementales sobre productos existentes}

Una de las relaciones más evidentes acerca de las consecuencias ambientales del uso del vehículo particular, es la emisión de gases contaminantes en su fase de uso. Es por eso que una de las aproximaciones más difundidas ha sido el diseño de productos más verdes en relación al combustible usado. Se trata de innovaciones incrementales donde se realizan mejorías a los productos existentes para disminuir su impacto ambiental. Es así que en la industria automotriz se han puesto grandes esfuerzos en la gestión del combustible y el uso de energías más limpias.

Desde una perspectiva de ciclo de vida, la mejora ambiental sólo se refleja en la etapa de uso, pudiendo contrariamente empeorar en etapas de disposición final. Este nivel de proyecto no requiere de cambios de comportamiento por parte de los usuarios y es general el resultado de iniciativas descendentes (top-down), relacionadas modelos capitalistas bajo el propósito de vender nuevos productos. Un ejemplo podrían ser los automóviles híbridos, eléctricos, etc. Se entiende que el lanzamiento de un producto más verde, en la mayoría de los casos, crea nuevas oportunidades de consumo, perdiendo todo sentido la estrategia en relación al uso eficiente de recursos.

Sin embargo, es importante mencionar que sí existen iniciativas incrementales a nivel producto que reflejan una mejoría radical en las prestaciones del mismo. En el campo de la movilidad existen proyectos que expanden la funcionalidad a nuevos ámbitos, como por ejemplo están las bicicletas plegables, donde una mejora incremental permite el uso de las mismas en circunstancias que antes eran limitantes. 


\section{b. La propuesta de productos nuevos (más sustentables)}

Este nivel implica el desarrollo y comercialización de productos que antes no estaban disponibles, concebidos en base a su eficiencia ambiental en todas las etapas de su ciclo de vida. Nuevos productos han sido propuestos para promover modos más sostenibles de desplazamiento, desde uniciclos a zapatos rodantes.

A pesar de que la innovación sucede mayormente en la esfera técnica, estas propuestas tienden a tener más dificultad para encajar en los patrones de consumo existentes ya que requieren de pequeños cambios en el contexto de consumo (Vezzoli, Manzini, 2008). Un ejemplo notorio en movilidad podría ser un dínamo modular que transforma las bicicletas normales en bicicletas eléctricas, haciendo así el mejor uso de los recursos existentes, ya que la innovación no requiere de que la persona cambie su bicicleta existente por una nueva. El consumo de estos productos significaría una mejora ambiental en la forma de satisfacer necesidades, en relación a todo el ciclo de vida.

\section{c. La gestión estratégica de sistemas de Productos y Servicios}

Este nivel cambia el foco del diseño del producto mismo a la satisfacción de la necesidad que motiva la existencia del producto en sus niveles más básicos. La innovación se produce mayormente en las nuevas conexiones y acuerdos entre los actores del sistema (stakeholders). Para que estas propuestas sean efectivas, deben ser social y culturalmente apreciadas para lo cual las campañas de comunicación son fundamentales.

A nivel sistema, se pueden proponer innovaciones sustanciales a través de la gestión estratégica de diferentes ofertas de transporte combinadas. Materialmente, los resultados pueden ser no visibles o sólo estar representados por elementos de interfaz, como lo sería una tarjeta prepaga de transporte, o por elementos de comunicación virtual, como un app en el teléfono. La innovación yace en las múltiples posibilidades de rutas que se ofrecen al haber combinado económica, virtual o comunicacionalmente diferentes modos, aumentando así la cobertura en el espacio y la eficiencia. Mayor innovación sucede en el caso de que los usuarios participen co-creando la oferta, compartiendo información entre sí y usando sus propias capacidades para complementar los viajes.

Este nivel permite alta versatilidad en la oferta, empoderando al usuario al brindarle las herramientas para planificar la forma más eficiente de satisfacer su necesidad particular de desplazamiento. Como un ejemplo, se puede presentar un usuario que usa herramientas digitales para planificar su viaje al trabajo y en base a clima, propósito del viaje y su estado de salud, ese día elige la estrategia de acceder en bicicleta, combinarla con TP y caminar el último tramo. Sin embargo, otro día que necesite realizar un viaje de compras podría elegir un automóvil compartido, o un taxi para ir al médico un día que se sienta enfermo.

\section{d. La propuesta de alternativas inteligentes a la posesión de vehículos}

El uso de vehículos particulares ha sido altamente estigmatizado en relación a políticas sostenibles de transporte, hecho que ha impulsado políticas de limitación de este modo en centros urbanos compactos y bien servidos por modos públicos. Sin embargo, existen tipologías de ciudades donde el vehículo particular posee un rol fundamental como ha- 
bilitante de movilidad, asimismo, existen esferas socio-económicas y propósitos de viajes donde no es realista esperar el abandono voluntario de estos modos. Estas brechas se pueden pasando de un modelo de posesión de producto a un modelo de acceso a un servicio. Si nos movemos desde una economía basada en los productos a una economía basada en los servicios, una estrategia creciente a favor de la sostenibilidad es la servificación. La economía de los servicios se ocupa de la optimización del uso (o performance) de bienes y servicios, gestionando los recursos ya existentes (bienes, conocimiento, naturaleza).

En este ámbito, los servicios de transporte tradicionales se han diversificado pasando por múltiples combinaciones entre individual, compartido, público y privado. En las últimas décadas, el cambio de paradigma ha comenzado a materializarse, y hasta compañías tradicionales (como Peugeot) han explorado los ámbitos de uso compartido, como el car sharing, como expansión estratégica de su modelo de negocios.

Los beneficios ambientales de la servificación encuentran no sólo en la extensión de vida del producto y el aumento de la intensidad de uso (al ser compartido). Se debe considerar el fin de la obsolescencia programada, si en vez de ser propietario de un vehículo, la persona lo alquila, el productor posee el incentivo económico de diseñar productos que sean durables, reparables, re-usables, actualizables y de bajo mantenimiento (Dooley, 2013).

El uso compartido de vehículos privados. Un tipo de movilidad que ha crecido radicalmente en la última década merece un análisis profundo, se trata de los servicios de taxi que utilizan aplicaciones basadas en la localización geográfica. Mediante estos servicios, los usuarios pueden contratar un vehículo con chofer a demanda (y compartir el viaje o no) pagando menos que el costo de un taxi tradicional. Probablemente el ejemplo más interesante es el de Uber.

Esta clase de servicios poseen menor impacto ambiental que los taxis tradicionales al hacer un uso más eficiente de los recursos. Mediante aplicaciones inteligentes se gestionan oferta y demanda en base a la locación, enviando el automóvil más cercano al cliente, ahorrando tiempo y recursos. Los clientes pueden elegir compartir el viaje (con el incentivo de compartir el gasto) para lo cual la app agrupa a los clientes que van en la misma dirección y les sugiere viajar juntos, reduciendo las cargas ambientales (asignando CO $2 / \mathrm{km} /$ persona). Socialmente, aspectos importantes como la seguridad personal de los viajantes han sido exitosamente gestionados mediante sistemas de calificación. Los pasajeros evalúan al conductor en cada viaje (los conductores dependen en las calificaciones para conseguir mayor cantidad de viajes) y de la misma forma, los conductores evalúan a los pasajeros (así los trabajadores de la empresa están protegidos). Se intenta promover los buenos tratos para mejorar el servicio y es así que se van reforzando las comunidades en la escala humana. La sostenibilidad social también se refleja en que los empleadores ofrecen la posibilidad a los empleados de manejar su horario de trabajo de forma flexible y voluntaria como contratistas independientes. Es radicalmente innovador el slogan "conduce tu propio auto y sé tu propio jefe" (Uber, 2017). Esta flexibilidad favorece la inclusión de grupos vulnerables que están desempleados temporalmente o que poseen dificultad en comprometerse a horarios fijos (como madres solteras o pacientes crónicos).

La transición hacia estos modelos ha sido difícil ya que la mayoría de los países no poseen legislación adecuada a este tipo de oferta, porque no encajan claramente en los modelos 
pre-establecidos. Al operar entre los límites de lo público y lo privado, las externalidades como responsabilidades legales en caso de accidente han debido redefinirse. Sumado a esto, los proveedores y los clientes han debido ser educados en el uso de esta nueva tipología de oferta, donde la comunicación estratégica, el diseño y la experiencia de usuario han jugado un rol clave.

Probablemente la mayor barrera de transición en Latinoamérica, ha sido la oposición gremial de quienes han perdido el control del mercado al enfrentarse con un competidor que ofrecía niveles de servicio más altos en precio y experiencia personal. En este marco, se debe reconocer que los servicios como Uber han abierto el camino a estándares más altos de calidad basados en necesidades reales, impulsando a que proveedores tradicionales mejoren su oferta para quedar en el mercado.

La innovación en este área está apenas comenzando, y numerosos grupos surgen cada día con modelos de negocios híbridos donde los consumidores son creadores y proveedores a la vez. Uno de los hechos que ha permitido quiebre radical es el uso de nuevas tecnologías y la diseminación de conocimientos en la red.

\section{e. La propuesta de nuevos escenarios de estilos de vida sostenibles}

El Diseño para la Transición (Transition Design) es una disciplina que propone la formulación de visiones de futuro que sean el resultado de innovación local ascendente (bottom$u p)$. Esta formulación de escenarios futuros es un proceso en constante evolución, que va aprendiendo de sus errores y adaptándose a los cambios culturales y contextuales. La sostenibilidad se logra mediante las elecciones de estilos de vida subsecuentes que han sido impulsadas por la visión, al crear espacios de debate acerca de nuevas formas de ser $y$ de hacer. La visualización ayuda a que los usuarios salgan del escepticismo y se pregunten acerca de cómo podrían ser las cosas (Dunne y Raby, 2013). Una vez establecida una visión de futuro adecuada, se puede planificar la transición del modelo actual al futuro, diseñando cada paso.

Estos escenarios de movilidad operan en la esfera cultural y se basan en la investigación, la colección y la re-interpretación de casos prometedores existentes, lo cual los hace realistas. Este nivel de proyecto reconoce la naturaleza trasversal de la problemática y sus raíces sociales, intentando resolver no sólo sus aspectos ambientales sino también los económicos y culturales (Barón, 2007). En el ámbito de la movilidad, a continuación se ha teorizado en la forma que es escenario SLOC (Manzini, 2009) podría materializarse.

El escenario Pequeño, Local, Abierto y Conectado. Ezio Manzini forjó el concepto de SLOC (significando Small, Local, Open and Connected, por sus siglas en inglés) con el propósito de sintetizar sus investigaciones en relación al diseño para la Innovación Social. Estableciendo que "La única forma sustentable de salir de la crisis financiera y ecológica global es la promoción de nuevos modelos económicos, nuevos sistemas de producción y nuevas ideas de bienestar". El modelo SLOC se ha convertido en una ley universal e inteligible que hace que cualquier proyecto sea viable y sostenible social, ambiental y económicamente (Manzini, 2009). 
SLOC, en términos generales, se refiere a un sistema de producción y consumo distribuido (pero conectado) donde la pequeña escala de cada nodo hace que sea comprensible, controlable, democrático, resiliente y altamente relacionado con el contexto. La práctica de visualizar escenarios bajo esta lupa, le brinda al diseñador indicios para definir caminos de transición.

Desde proyectos de Innovación Social al Placemaking, cientos de proyectos de movilidad, exitosos en la pequeña escala, están disponibles en internet para ser estudiados e interpretados. Los ejemplos son diversos: desde grupos de Walking-Bus (grupos de niños supervisados por un adulto voluntario en su camino peatonal hacia la escuela) que refuerzan una rutina diaria sana y socialmente inclusiva, hasta clubes de reparación de bicicletas, urbanismo de guerrilla, car-pooling, etc. (Meroni, 2007).

Estos proyectos individualmente pueden diferir en su forma de ejecución pero poseen características comunes que brindan pistas acerca de cómo puede materializarse la transición. En general, todos desafían los paradigmas de movilidad existentes y representan cambios radicales en la escala local. Muestran que el tiempo, el dinero y las relaciones sociales se pueden medir de forma diferente en relación a la calidad de vida. Es por esto que sirven como laboratorios de futuros posibles, donde en cada caso un grupo de individuos altamente innovadores han exitosamente sus hábitos de movilidad, consumiendo menos recursos naturales, incrementando al mismo tiempo su nivel de bienestar y fortaleciendo la comunidad. Cada iniciativa puede parecer demasiado pequeña para hacer una diferencia, pero juntas, conectadas, son más grandes y más fuertes, brindando inspiración y estimulando el imaginario colectivo, rompiendo la inercia de la mayoría hacia cambios significativos.

\section{Conclusiones}

¿Cuál es, por lo tanto, movilidad urbana sostenible? Al lidiar con sistemas complejos, no existe una respuesta única a esta pregunta. De hecho, sería un gran error pretender que la hubiera. Sin embargo, se identifican palabras clave que pueden describir a las posibles soluciones. La movilidad sostenible debería ser: centrada en las personas, versátil, limpia, participativa, transparente, eficiente, inclusiva, contextual, diversa, abierta, errorfriendly...

Probablemente esto no es nada nuevo para el lector, ya que estas palabras se han utilizado numerosas veces para proponer soluciones a cualquier problemática trasversal global. A pesar de esto, es llamativo que muchos tomadores de decisiones siguen invirtiendo fondos públicos para subsidiar propuestas débiles (como por ejemplo la industria de automóviles eléctricos) en base a visiones limitadas de la problemática que no operan en niveles sociales y culturales de re-definición de hábitos.

La visión a futuro de las propuestas más sustentables se basa en un sistema privado/público, compuesto por diferentes tipos de vehículos, aquellos motorizados usando las energías más limpias disponibles en cada contexto. Se trataría de un sistema económicamente accesible, inteligente en relación al uso de nuevas tecnologías, especialmente aquellas de comunicación con los usuarios, con plataformas participativas en tiempo real. Un sistema co-diseñado, que aprenda de errores y valore la información generada por usuarios, que se 
adapte a cambios contextuales. Este sistema debería estar inmerso en un contexto urbano compacto y con usos del suelo mixtos. Un contexto seguro, inclusivo, vibrante y hermoso. Una ciudad con excelente infraestructura ciclista y peatonal segura, con regulaciones de velocidad adecuadas para los vehículos motorizados. Una ciudad donde los viajes necesarios sean aquellos electivos, con opciones de teletrabajo y la posibilidad de realizar trámites de forma remota.

Si analizamos este escenario futuro, innovador y radical, nos damos cuenta de que actualmente contamos con todas las herramientas y tecnologías necesarias para materializarlo. De hecho los únicos cambios materiales necesarios son aquellos de índole espacial, urbana, manteniéndose constante la oferta de productos y servicios. La innovación está dada por la gestión eficiente, inteligente y participativa de los recursos. Y más radicalmente por las nuevas concepciones acerca del bienestar, que se alejan de las propuestas por el mercado y se acercan a aquellas innatas a las personas. Se trata de una propuesta centrada en el usuario no sólo a nivel de escala física, sino a escala emocional y cognitiva.

En síntesis, los decisores deben dejar de priorizar las soluciones técnicas por sobre las culturales. Esta problemática urbana, trasversal a todas las actividades humanas, posee profundas raíces políticas y culturales que albergan el mayor potencial de innovación. Se debe trabajar sobre nuevos modelos de bienestar urbano, con criterios de calidad basados en el uso del tiempo, las relaciones sociales y la salud, donde la movilidad se asocie experiencias placenteras.

El Diseño es una disciplina que puede jugar un rol clave en esta transición, desde la propuesta de escenarios alternativos viables, atractivos e inteligibles hasta la planificación estratégica y participativa de su implementación gradual. Tal vez se necesiten nuevos productos, pero la innovación radical será inmaterial, revolucionando sistemas sociales, económicos y políticos, mediante la introducción ascendente de nuevas formas de ser y de hacer.

\section{Referencias}

Borst, H. C., de Vries, S. I., Graham, J. M. A., van Dongen, J. E. F., Bakker, I. \& Miedema, H. M. E. (2009). Influence of environmental street characteristics on walking route choice of elderly people. Journal of Environmental Psychology, 29(4), 477-484.

Capra, Fritjof and Pier Luigi Luisi, (2014). The Systems View of Life: A Unifying Vision. Padstow, Cornwall: Cambridge University Press.

Charter of Public Space (2013). Final session of the 2013 Biennial of Public Space. Rome.

Culén, A., Velden, M. Van Der, \& Herstad, J. (2014). Travel experience cards: capturing user experiences in public transportation. ACHI 2014: The Seventh Internatinal Conference on Advances in Computer-Human Interactions, (c), 72-78.

Dooley, K. (2013). Product design: Do it with Dematerialization. CSR Wire, 14-16. Retrieved from: http://www.csrwire.com/blog/posts/1078-product-design-do-it-withdematerialization

Dunne, A. and Raby, F. (2013). Speculative Everything: Design, Fiction, and Social Dreaming, pp. 1-9. Cambridge, MA: The MIT Press. 
Gomez, L. M. (2000). Gender Analysis of Two Components of the World Bank Transport Projects in Lima, Peru: Bikepaths and Busways. World Bank Internal Report, World Bank, Washington, D.C.

Gutiérrez, A. (2010). Movilidad, transporte y acceso: una renovación aplicada al ordenamiento territorial. En: Scripta Nova, vol. XIV, No. 331 (86). Barcelona: Universidad Autónoma de Barcelona. En: www.ub.edu/geocrit/sn/sn-331/sn-331-86.html

Gutiérrez, A. (2012). ¿Qué Es La Movilidad?. Bitácora 2121 (2): 61-74.

Gutiérrez, A., Rearte, J. (2010) Movilidad y centralidad. Reflexiones entorno al debate sobre la nueva estructura urbana y el ordenamiento territorial. CODATU XIV. Buenos Aires.

Hernández, D. (2012). Activos y estructuras de oportunidades de movilidad. Una propuesta analítica para el estudio de la accesibilidad por transporte público, el bienestar y la equidad. En: EURE Revista Latinoamericana de Estudios Urbano Regionales, vol. 38, No. 115. Santiago de Chile: Pontificia Universidad Católica de Chile.

Handy, S. (1996). Methodologies for exploring the link between urban form and travel behavior, 1(2), 151-165. Transpn Res. D, Vol. 1, No. 2, pp. 151-165. Elsevier Science, Great Britain.

Hsiao, S., Lu, J., Sterling, J., \& Weatherford, M. (1997). Use of geographic information system for analysis of transit pedestrian access. Transportation Research Record, 1604, 50-59.

IVM - Institute pour la ville en movement (2013) Legible City, Ciudad Legible. Obtenido de la página www.ciudadenmovimiento.org en Mayo de 2015.

Kaufmann, v. (2002). Re thinking mobility. Aldershot Ashgate.

Kuby, M., Barranda, A., \& Upchurch., C. (2004). Factors influencing light rail station boardings in the United States. Transportation Research Part A, 38, 223-247

Lam, W., \& Morrall, J. (1982). Bus passenger walking distances and waiting times: A summerwinter comparison. Transportation Quarterly, 36(3), 407-421.

Lévy, J. (2001). “Os novos espacos da mobilidade”. En: Geographia, vol. 3, No. 6. Quito: Revista de la Orga- nización Latinoamericana y del Caribe de Centros Históricos.

Lindón, A. (2011) "Revisitar la concepción de lo social para una Geografía constructivista”. En: Zusman, P.; Haesbaert, R.; Castro, H. Y Adamo, S. (ed.) Geo- grafías culturales. Aproximaciones, intersecciones y desafíos. Buenos Aires: Ed. FFyL - UBA.

Litman, T. (2008). Sustainable Transportation Indicators. Transportation Research Board Annual Meeting 2009.

Manzini, E. (2009). An orienting scenario for social innovation and design in the age of networks. Ezio Manzini Public Lecture Lecture. Institute for Advanced Studies. Glasgow.

Martin, R. L. (2009). Design of business: Why design thinking is the next competitive advantage. Harvard Business Press.

Max-Neef, Manfred A. (1992). Human Scale Development: Conception, Application and Further Reflections. New York: Apex.

Meroni, A. (2007) Creative communities, Edizioni POLIdesign. Milano.

Miralles-Guasch, C. (2012). "Las encuestas de movilidad y los referentes ambientales de los transportes”. En: EURE Revista Latinoamericana de Estudios Urbano Regionales, vol. 38, No. 115. Santiago de Chile: Pontificia Universidad Católica de Chile.

Murray, A., Davis, R., Stimson, R., \& Ferreira, L. (1998). Public transportation access. Transportation Research Part D, 3(5), 319-328. 
Miettinen, S. and Koivisto, M. (2009). Designing Services with Innovative Methods. Univ. of Art and Design Helsinki.

Project for Public Spaces (2012). Placemaking and the future of cities. DRAFT. Produced under the auspices of the UN-HABITAT Sustainable Urban Development Network (SUD-Net).

Stahel W. (2001). Sustainability and Services, in Sustainable Solutions - Developing products and services for the future, Charter M. and Tischner U. (edit by), Greenleaf publishing, UK.

Tukker, A. (2004). Eight types of product-service system: eight ways to sustainability? Experiences from suspronet, Business Strategy and the Environment, vol. 13, no. 4

Vezzoli, C. (2007). System Design for Sustainability. Maggioli Editore, Milano, Italy.

Vezzoli, C., \& Manzini, E. (2008). Design for Environmental Sustainability. London: Springer London. http://doi.org/10.1007/978-1-84800-163-3.

Orfeuil, J. P. (dir.) (2004). Transports, pauvretés, exclusions. Pouvoir bouger pour s'en sortir. Paris: éditions de L'Aube.

O'Sullivan, S., Morrall, J. (1996). Walking distance to and from light-rail transit stations. Transportation Research Record, 1538, 19-26.

Papanek, V. (1983). Design for Human Scale, New York, Van Nostrand Reinhold. ISBN 0-442-27616-8.

Pucher, J., Buehler, R. (2008) Making Cycling Irresistible: Lessons from Making Cycling Irresistible, 28(4)

Schlossberg, M., Agrawal, A., Irvin, K., \& Bekkouche, V. (2007). How far, by which route, and why? A spatial analysis of pedestrian preference. MTI Report 06-06. San José, CA: Mineta Transportation Institute \& College of Business, San José State University.

Shakespear, Ronald (2014). Haciendo La Ciudad Legible. Instituto para la ciudad en movimiento. Buenos Aires. Disponible en http://ciudadenmovimiento.org . Consultado en Junio de 2016.

Thompson, S. R., Monsere, C. M., Figliozzi, M., Koonce, P., \& Obery, G. (2013). BicycleSpecific Traffic Signals. Transportation Research Record: Journal of the Transportation Research Board, 2387(-1), 1-9. doi:10.3141/2387-01

Valenzuela-Montes, L., Talavera-García, R. (2015). Entornos de Movilidad Peatonal: Una Revisión de Enfoques, Factores Y Condicionantes. EURE. Revista Latinoamericana de Estudios Urbanos Regionales 41 (123): 5-27. doi:10.4067/S0250-71612015000300001.

Wardman, M. (1998) The Value of Travel Time: A Review of British Evidence (1998) Journal of Transport Economics and Policy Vol. 32, No. 3, pp. 285-316.

World Bank. (2002). Cities on the move. A World Bank Urban Transport Strategy Review. Washington, D.C.

Zacharias, J. (2001). Pedestrian Behavior and Perception in Urban Walking Environments. Journal of Planning Literature, 16(1), 3-18. doi: 10.1177/08854120122093249

Abstract: Transition Design is a discipline that proposes the formulation of future visions that are the result of upward local innovation in constant evolution. In relation to the problem of urban mobility, sustainability is achieved through the choices of lifestyles that 
have been driven by the vision, by creating spaces for debate about new ways of being and doing, and by co-designing strategies to materialize that vision.

In the field of sustainable mobility, innovation does not need to be of a material nature, but rather results from the efficient, intelligent and participatory management of resources. It is radical in its conceptions about well-being, that move away from those proposed by the market and approach those innate to people. It is a mobility on a human scale, centered on the user not only at the level of physical scale, but on an emotional and cognitive scale. The following work presents a literature review on mobility as a transversal activity to all urban activities, proposing the reduction to the human scale as a strategy for the transition. Finally, a possible scenario of sustainable mobility is proposed, where the role of the designer as a cultural operator becomes fundamental.

Key words: sustainable mobility - public space - walking - cycling - social innovation participatory design - urban welfare.

Resumo: O Design para a transicao é uma disciplina que propõe a formulação de visões futuras que são o resultado da inovação local ascendente em constante evolução. Em relação ao problema da mobilidade urbana, a sustentabilidade é alcançada através das escolhas de estilos de vida que foram conduzidas pela visão, criando espaços para o debate sobre novas formas de ser e de fazer, e por co-conceber estratégias para materializar essa visão.

No campo da mobilidade sustentável, a inovação não precisa ser de natureza material, mas sim da gestão eficiente, inteligente e participativa dos recursos. É radical em suas concepções sobre o bem-estar, que se afastam daqueles propostos pelo mercado e abordam os inatos para as pessoas. É uma mobilidade em escala humana, centrada no usuário não só ao nível da escala física, mas em uma escala emocional e cognitiva. $\mathrm{O}$ trabalho a seguir apresenta uma revisão da literatura sobre a mobilidade como atividade transversal para todas as atividades urbanas, propondo a redução da escala humana como estratégia para a transição. Finalmente, propõe-se um cenário viável de mobilidade sustentável, onde o papel do designer como operador cultural se torna fundamental.

Palavras chave: mobilidade sustentável - espaço público - caminhada - ciclismo - inovação social - design participativo - bem-estar urbano. 